\title{
Como a Realidade Aumentada tem Auxiliado no Processo de Ensino e Aprendizagem de Ciências da Natureza? Um Mapeamento Sistemático da Literatura
}

\author{
Eduarda Queiroz1, Rafaela Moura1, Ellen Souza1 \\ 1Universidade Federal Rural de Pernambuco - Unidade Acadêmica de Serra Talhada \\ (UFRPE - UAST) - Serra Talhada, PE - Brasil \\ mequeirozs@gmail.com, rafaela.smm98@gmail.com, ellen.ramos@ufrpe.br
}

\begin{abstract}
Augmented Reality (AR) is defined as any system that combines the real with the virtual, which is interactive in real time and is registered in three dimensions. In the teaching of the natural sciences it can provide students with the visualization of abstract concepts. This work aims to investigate how RA has aided in the teaching and learning process of the natural sciences. A systematic mapping was carried out, which is a secondary study and aims to identify the research gaps in the literature. Primary studies published between the years of 2009 to 2018 were analyzed in order to extract data that could answer questions and thus reach the objective of this work.
\end{abstract}

Resumo. A Realidade Aumentada (RA) é definida como qualquer sistema que combine o real com o virtual, que seja interativo em tempo real e que esteja registrado em três dimensões. No ensino das ciências da natureza, ela pode proporcionar aos estudantes a visualização de conceitos abstratos. Este trabalho tem como objetivo investigar como a RA tem auxiliado no processo de ensino e aprendizagem das ciências naturais. Foi realizado um mapeamento sistemático que é um estudo secundário e tem como foco identificar as lacunas de pesquisas na literatura. Estudos primários publicados entre os anos de 2009 a 2018 foram analisados a fim de extrair dados que pudessem responder às questões de pesquisa e assim alcançar o objetivo deste trabalho.

\section{Introdução}

Em 2015, os resultados do Programa Internacional de Avaliação dos Estudantes (PISA) mostraram que o Brasil ocupa a $63^{\mathrm{a}}$ posição em Ciências, área esta que foi o foco da prova naquele ano (PISA, 2018). Este dado mostra que há uma deficiência no aprendizado das disciplinas correspondentes às ciências naturais. Esta deficiência se dá porque grande parte dos professores adotam um ensino baseado na transmissãorecepção de conhecimento, nos qual eles são os agentes ativos no processo e, como consequência, a aprendizagem é caracterizada pela memorização, que resulta em um esquecimento rápido do que foi "ensinado" (Schnetzler, 1992, p. 17). Valente (2018) evidencia que é necessário alterar a maneira como os conteúdos disciplinares são trabalhados e que a sala de aula deve ter uma dinâmica coerente com o dia a dia dos estudantes, cada vez mais mediado pelas tecnologias digitais. 
A Realidade Aumentada (RA) é definida como qualquer sistema que combine o real com o virtual, que seja interativo em tempo real e que esteja registrado em três dimensões. A RA permite ao usuário ver o mundo real sobreposto ou composto por objetos virtuais. Nesse tipo de sistema, o real e o virtual coexistem no mesmo espaço. Ela suplementa a realidade (Azuma, 1997). A educação é uma área, em especial, na qual esta tecnologia pode ser valiosa, pois, de uma perspectiva pedagógica, a atividade experimental baseada na observação prática de conceitos proporciona uma conexão entre a teoria e a realidade, enriquecendo e solidificando o conhecimento teórico (Gruber et al., 2011). A RA proporciona uma experiência educacional diferente, como: um comportamento conversacional face a face, onde os alunos podem sentar em volta de uma mesa, vendo e interagindo uns com os outros ao mesmo tempo que com um coração flutuante; uma interface tangível, com a qual até mesmo crianças muito jovens podem ter uma experiência rica com a RA; e uma interface transitória, onde os usuários podem fazer uma transição suave do estático para o interativo (Billinghurst, 2002). No ensino das ciências naturais, ela pode proporcionar aos estudantes a visualização de conceitos abstratos como, por exemplo, os movimentos ondulatórios na física.

Sendo assim, este trabalho tem como objetivo investigar como a realidade aumentada tem auxiliado no processo de ensino e aprendizagem de ciências da natureza. Para a realização do mesmo, foi realizado um Mapeamento Sistemático da Literatura (MSL), um tipo de estudo secundário, utilizado para identificar as lacunas de pesquisas na literatura a partir da análise e extração de dados de estudos primários, sendo possível, assim, identificar em quais áreas temáticas e para quais tipos de pesquisas há escassez de publicações (PETERSEN, 2008). Na Seção 2, são apresentados os trabalhos relacionados a este mapeamento. A Seção 3 expõe o método utilizado para construir este trabalho. Na Seção 4, são apresentados os resultados. A Seção 5 desenvolve a discussão sobre os resultados deste trabalho. Por fim, a Seção 6 apresenta a conclusão deste artigo.

\section{Trabalhos Relacionados}

Nesta seção são apresentados os trabalhos relacionados, abordando os objetivos, o método e resultados dos mesmos. Ao final, os estudos apresentados são comparados.

\subsection{Revisão Sistemática sobre Jogos Digitais que utilizam Realidade Aumentada no Contexto Educacional Brasileiro}

Oliveira et al. (2014) analisaram 872 estudos primários retirados dos Anais Eletrônicos do SBGames (evento da Sociedade Brasileira de Computação - SBC e da Abragames Associação Brasileira dos Desenvolvedores de Jogos Digitais) no período de 2006 a 2013, com o objetivo de conhecer como os pesquisadores brasileiros têm feito uso da Realidade Aumentada em jogos educacionais. Primeiramente, foram definidas as questões de pesquisa. Em seguida, foi realizada a análise dos estudos através de uma ferramenta automatizada desenvolvida para extração de dados que utilizava como palavras-chave os termos: "virtual reality", "realidade virtual", "augmented reality", "realidade aumentada", "education" e "educação". Os dados obtidos foram organizados em uma tabela para que pudessem ser interpretados a fim de responder às questões de pesquisa. Ao final, os autores perceberam que em mais da metade da amostra total não foram encontrados nenhum dos termos; que $2 \%$ da amostra estava relacionada a RA e 
que somente $0,6 \%$, aproximadamente, relacionava RA à educação, evidenciando assim que esta é uma área a ser mais explorada pelos cientistas no Brasil.

\subsection{Revisão Sistemática sobre Jogos Digitais que utilizam Realidade Aumentada no Contexto Educacional Brasileiro}

Moro (2018) analisou 2111 estudos primários retirados do Google Acadêmico, IEEE, Scopus, Springer, Science Direct, Ebsco e ACM, no período de 2012 a 2017, dos quais 21 estudos foram incluídos em sua revisão, com o objetivo de apresentar pesquisas e aplicações de RA em livros infantis. Se baseando nas etapas de planejamento, execução e extração de Kitchenham \& Charters (2007) e adaptadas de Neiva e Silva (2016), foram definidas as questões principal e as específicas, como também foram iniciadas as buscas. Na execução, foi realizado um refinamento dos estudos retornados com base em critérios de inclusão ou exclusão das pesquisas. Finalmente, na extração, foram coletados e sintetizados os dados dos trabalhos selecionados que passaram por análises mais específicas pretendendo, assim, responder às questões definidas no planejamento da revisão. Ao final, a autora constatou que o uso da RA pode facilitar a compreensão da história e aproximar a criança de aspectos mais complexos.

O Quadro 1 apresenta uma análise e comparação entre os estudos retratados acima e este mapeamento baseada no período de busca, tipo de busca, ambiente de atuação e fontes de busca. Em divergência com os trabalhos relacionados, este utilizou oito fontes de pesquisa, realizou dois tipos de buscas e teve um período de pesquisa compreendido em dez anos.

\begin{tabular}{|c|c|c|c|c|}
\hline Autor(es) & Período & Tipo de Busca & $\begin{array}{c}\text { Ambiente de } \\
\text { Atuação }\end{array}$ & Fontes de Busca \\
\hline $\begin{array}{c}\text { Oliveira et al. } \\
\text { (2014) }\end{array}$ & 2006 a 2013 & Manual & Educacional & $\begin{array}{c}\text { Anais Eletrônicos do } \\
\text { SBGames }\end{array}$ \\
\hline Moro (2018) & 2012 a 2017 & Automática & Educacional & $\begin{array}{c}\text { Google Acadêmico, } \\
\text { IEEE, Scopus, Springer, } \\
\text { Science Direct, Ebsco e } \\
\text { ACM }\end{array}$ \\
\hline Este trabalho & 2009 a 2018 & $\begin{array}{c}\text { Manual e } \\
\text { automática }\end{array}$ & Educacional & $\begin{array}{c}\text { CBIE, WIE, RBIE, } \\
\text { RENOTE, JAIE, SBIE, } \\
\text { CTRL+E, Portal de } \\
\text { Periódicos CAPES }\end{array}$ \\
\hline
\end{tabular}

Fonte: Elaborado pelos Autores (2019)

\section{Método}

Nesta pesquisa, realizou-se um mapeamento sistemático da literatura, baseado no guia proposto por Petersen et al. (2008). Este guia está organizado em 5 etapas, como mostra na Figura 1. (I) Definição do Protocolo de Pesquisa, (II) Condução da Busca, (III) PréSeleção, (IV) Seleção e (V) Extração dos Dados.

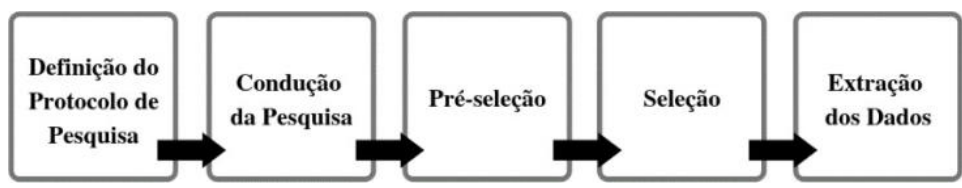


Figura 1. Etapas do mapeamento. Fonte: Adaptada de Petersen et al. (2008)

\subsection{Protocolo de Pesquisa}

Nesta etapa foi elaborado o protocolo do mapeamento sistemático, começando pela definição do tema central e dando continuidade com as questões de pesquisa, fontes e strings de busca e, por fim, os critérios de inclusão e exclusão, como segue nas subseções abaixo.

\subsubsection{Questões de Pesquisa}

Para alcançar o objetivo do mapeamento, foram elaboradas uma questão principal (QP) e questões específicas $(\mathrm{QE})$ a serem respondidas e que estão apresentadas abaixo.

"QP - Como a Realidade Aumentada tem Auxiliado no Processo de Ensino e Aprendizagem de Ciências da Natureza?"

- QE1 - Qual a evolução total e por disciplina, de estudos primários de 2009 a 2018 ?

- QE2 - Quais autores, regiões, estados e instituições relatam o uso de RA para o ensino de ciências da natureza?

- QE3 - Quais tecnologias estão disponíveis para o ensino de ciências da natureza com RA?

- QE4 - Houve avaliação quanto ao uso de RA no ensino?

- QE5 - Em quais níveis de ensino a RA foi usada para lecionar ciências naturais?

\subsubsection{Seleção de Fontes e String de Busca}

$\mathrm{Na}$ busca manual, elegeu-se revistas e eventos de destaque na área de informática na educação, apresentados no Quadro 2. Na busca automática, foi selecionado o Portal de Periódicos CAPES, o qual indexa uma biblioteca digital de relevância na academia, utilizando a string de busca apresentada no mesmo quadro, logo abaixo do nome do portal.

Quadro 2. Fontes de Busca

\begin{tabular}{|c|}
\hline Manual \\
\hline Congresso Brasileiro de Informática na Educação (CBIE). \\
\hline Workshop de Informática na Escola (WIE). \\
\hline Congresso sobre Tecnologias na Educação (Ctrl+E). \\
\hline Simpósio Brasileiro de Informática na Educação (SBIE). \\
\hline Revista Brasileira de Informática na Educação (RBIE). \\
\hline Revista Novas Tecnologias na Educação (RENOTE). \\
\hline Jornada de Atualização em Informática na Educação (JAIE). \\
\hline Automática \\
\hline Portal de Periódicos CAPES \\
String de Busca: "realidade aumentada" AND ("física" OR "química" OR "biologia") \\
\hline
\end{tabular}

Fonte: Elaborado pelos Autores (2019)

\subsubsection{Critérios de Inclusão e Exclusão}


Diante da proposta do MSL, foram definidos critérios de inclusão (CI) e de exclusão (CE). Como mostra o Quadro 3, abaixo.

\begin{tabular}{|l|l|}
\multicolumn{2}{|c}{ Quadro 3. Critério de Inclusão e Exclusão } \\
\hline $\begin{array}{l}\text { Critérios de Inclusão } \\
\begin{array}{l}\text { C11 - Estudos primários publicados entre } \\
2009 \text { e 2018. }\end{array}\end{array}$ & CE1 - Estudo primário duplicados. \\
\hline $\begin{array}{l}\text { Cl2 - Estudos primários publicados } \\
\text { em língua portuguesa. }\end{array}$ & $\begin{array}{l}\text { CE2 - Estudos secundários (revisões e } \\
\text { mapeamentos sistemáticos). }\end{array}$ \\
\hline $\begin{array}{l}\text { Cl3 - Estudos primários que estão } \\
\text { disponíveis online. }\end{array}$ & $\begin{array}{l}\text { CE3 - Estudos primários que não apresentem o } \\
\text { uso de realidade aumentada no ensino e } \\
\text { aprendizagem de ciências da natureza. }\end{array}$ \\
\hline
\end{tabular}

Fonte: Elaborado pelos Autores (2019)

\subsection{Pré-seleção}

Nesta etapa, foram analisados e catalogados os estudos primários retornados das buscas manual e automática que foram publicados entre os anos de 2009 a 2018. Os estudos foram organizados em uma tabela juntamente com os critérios que foram aplicados aos mesmos. Esta tabela está disponível no link1. Na catalogação, para a aplicação dos critérios, foram analisados os títulos, resumos, o tipo da publicação e primeiro autor de cada estudo. No final 21 trabalhos foram pré-selecionados como mostra no Quadro 4.

\subsection{Seleção}

Nesta etapa, os 21 estudos pré-selecionados foram analisados novamente, sendo que agora, de forma mais detalhada. A partir da leitura completa dos mesmos, foram extraídas as informações necessárias para que as questões de pesquisas pudessem ser respondidas.

\subsection{Condução da Busca}

Dentre os 4814 estudos analisados, 79 foram retornados na busca manual, após a execução da palavra-chave 'realidade aumentada'. Na busca automática, após a execução da string de busca, 18 estudos foram retornados. Em seguida, aplicou-se os critérios de inclusão e exclusão, resultando no total de 21 estudos incluídos na etapa de pré-seleção. Na etapa de seleção, realizou-se a leitura completa dos mesmos a fim de extrair dados que respondessem às questões de pesquisa. Os 21 estudos passaram no processo de seleção e foram incluídos no mapeamento, Quadro 4.

\begin{tabular}{|c|c|c|c|c|c|c|c|c|c|}
\multicolumn{7}{c|}{ Quadro 4. Resultados da condução da busca } & $\begin{array}{c}\text { Busca } \\
\text { Automática }\end{array}$ & \multirow{2}{*}{ TOTAL } \\
& \multicolumn{7}{|c|}{ Musca Manual } \\
\hline & CBIE & WIE & RBIE & RENOTE & JAIE & SBIE & CTRL+E & CAPES & \\
\hline Publicados & 926 & 723 & 256 & 1181 & 39 & 1471 & 218 & - & 4814 \\
\hline Retornados & 21 & 4 & 4 & 21 & 0 & 25 & 4 & 18 & 97 \\
\hline Pré-seleção & 5 & 1 & 2 & 6 & 0 & 3 & 1 & 3 & 21 \\
\hline
\end{tabular}




\begin{tabular}{|l|l|l|l|l|l|l|l|l|l|}
\hline Seleção & 5 & 1 & 2 & 6 & 0 & 3 & 1 & 3 & 21 \\
\hline
\end{tabular}

Fonte: Elaborado pelos Autores (2019)

\subsection{Extração dos Dados}

A fim de responder às questões de pesquisa apresentadas na Subseção 3.1.1, foram extraídos os seguintes dados: título do do estudo primário, tipo (artigo curto ou completo), primeiro autor, disciplina, autores, região, tecnologias disponíveis, gratuidade das tecnologias, status de desenvolvimento da tecnologia, avaliação do uso da tecnologia e nível de ensino onde o estudo foi aplicado. Foi utilizada uma tabela para organizar os dados coletados, que está disponível no link2.

\section{Resultados}

Nesta Seção, são apresentadas as respostas para as questões específicas de pesquisa. As mesmas foram obtidas a partir da extração dos dados dos 21 estudos primários considerados ao fim do mapeamento.

\subsection{QE1 - Qual a evolução total e por disciplina, de estudos primários de 2009 a 2018?}

A Figura 2 mostra que dos anos de 2009 a 2017 a quantidade de publicações foi relativamente baixa se comparadas ao ano de 2018. Logo, só houve uma evolução considerável no último ano compreendido pelo mapeamento. Observa-se também que, diante das disciplinas abordadas, a com maior número de aplicação foi física.

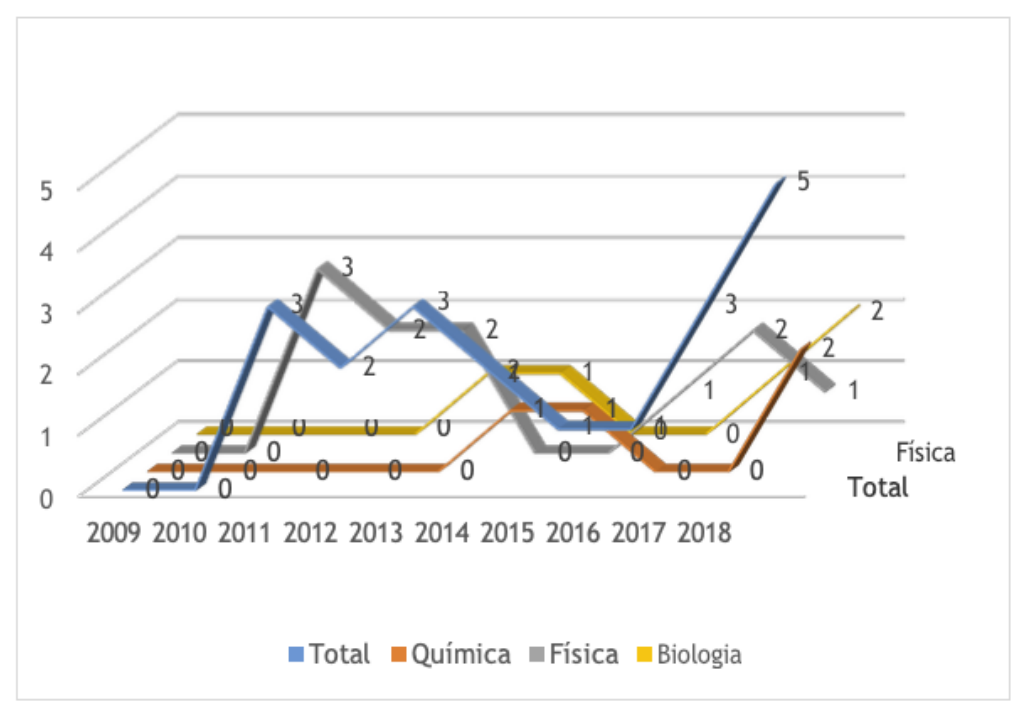

Figura 2. Evolução de estudos primários por disciplina. Fonte: Elaborada pelos autores (2019)

No Quadro 5, é apresentado o quantitativo de estudos por ano, em relação ao total e às disciplinas de química, física e biologia.

Quadro 5. Relação de estudos por ano 


\begin{tabular}{|c|c|c|c|c|c|c|c|c|}
\hline \multicolumn{2}{|c|}{ Ano } & \multicolumn{2}{|c|}{ Total } & \multicolumn{2}{c|}{$\begin{array}{c}\text { Disciplina de } \\
\text { Química }\end{array}$} & \multicolumn{2}{c|}{ Disciplina de Física } & \multicolumn{2}{c|}{ Disciplina de Biologia } \\
\cline { 2 - 10 } & Quant. & $\begin{array}{c}\text { Relação de } \\
\text { Estudos }\end{array}$ & Quant. & $\begin{array}{c}\text { Relação de } \\
\text { Estudos }\end{array}$ & Quant. & $\begin{array}{c}\text { Relação de } \\
\text { Estudos }\end{array}$ & Quant. & $\begin{array}{c}\text { Relação de } \\
\text { Estudos }\end{array}$ \\
\hline 2011 & 3 & EP9, EP10, EP15 & 0 & - & 3 & EP9, EP10, EP15 & 0 & - \\
\hline 2012 & 2 & EP7, EP16 & 0 & - & 2 & EP7, EP16 & 0 & - \\
\hline 2013 & 3 & EP1, EP8, EP11 & 0 & - & 2 & EP8, EP11 & 1 & EP1 \\
\hline 2014 & 2 & EP2, EP19 & 1 & EP2 & 0 & - & 1 & EP19 \\
\hline 2015 & 1 & EP20 & 1 & EP20 & 0 & - & 0 & - \\
\hline 2016 & 1 & EP12 & 0 & - & 1 & EP12 & 0 & - \\
\hline 2017 & 3 & EP3, EP6, EP13 & 0 & - & 2 & EP3, EP13 & 1 & EP6 \\
\hline 2018 & 5 & $\begin{array}{c}\text { EP4, EP5, EP14, } \\
\text { EP17, EP21 }\end{array}$ & 2 & EP4, EP21 & 1 & EP5 & 2 & EP17, EP18 \\
\hline
\end{tabular}

Fonte: Elaborado pelos Autores (2019)

\subsection{QE2 - Quais autores e regiões relatam o uso de RA para o ensino de ciências da natureza?}

Os autores com os números mais relevantes de publicações são: Suzana da Hora Macedo (IFF) com 5 estudos, Filipe Arantes Fernandes (IFF), Maria Cristina Villanova Biazus (UFRGS) e José Valdeni De Lima (UFRGS) com 4 artigos. Por fim, 7 autores com 2 estudos e outros 44 publicaram apenas 1 estudo. O Quadro 6 aponta que as regiões Sudeste e Sul apresentam um maior número de estudos sobre RA no ensino das ciências naturais.

Quadro 6. Relação de estudos por região

\begin{tabular}{|c|c|c|}
\hline Região & Quantidade & Relação de Estudos \\
\hline Sudeste & 12 & $\begin{array}{c}\text { EP1, EP4, EP7, EP9, EP10, EP12, EP13, EP15, EP16, EP17, EP19, } \\
\text { EP20 }\end{array}$ \\
\hline Sul & 12 & $\begin{array}{l}\text { EP2, EP3, EP5, EP6, EP8, EP9, EP11, EP12, EP13, EP14, EP15, } \\
\text { EP16 }\end{array}$ \\
\hline Norte & 2 & EP20, EP21 \\
\hline Nordeste & 2 & EP18, EP21 \\
\hline
\end{tabular}

Fonte: Elaborado pelos Autores (2019)

Somando a quantidade de estudos apresentados no Quadro 6, obtém-se um total de 28 estudos, que ultrapassa o número de 21 estudos selecionados e incluídos neste mapeamento. Isto se dá porque um mesmo artigo está afiliado a mais de uma instituição.

\subsection{QE3 - Quais tecnologias estão disponíveis para o ensino de ciências da natureza com RA?}

O tipo de tecnologia que obteve destaque quantitativo ao final deste estudo foram as aplicações móveis, com um total de 10 estudos apresentaram o desenvolvimento/uso de apps, conforme apresentando no Quadro 7. 
Quadro 7. Relação de estudos por tecnologias

\begin{tabular}{|c|c|c|}
\hline Tecnolgias & Quantidade & Relação de Estudos \\
\hline Aplicativos Móveis & 10 & EP2, EP3, EP4, EP5, EP6, EP12, EP13, EP18, EP19, \\
& 4 & EP21 \\
\hline Bibliotecas de RA & 4 & EP7, EP11, EP16, EP20 \\
\hline Ferramenta para RA & 4 & EP1 \\
\hline Sistema Desktop & 1 & EP14 \\
\hline Sistema Web & 1 & EP17 \\
\hline Página Web & 1 & \\
\hline
\end{tabular}

Fonte: Elaborado pelos Autores (2019)

Com relação ao status das tecnologias, 16 delas eram produtos finais, enquanto 5 eram protótipos. Quanto a acessibilidade das tecnologias, a maioria delas podem ser encontradas facilmente na internet ou em lojas virtuais de aplicativos de maneira gratuita.

\subsection{QE4 - Houve avaliação quanto ao uso de RA no ensino?}

Analisando os estudos selecionados, 11 estudos primários apresentaram avaliação das suas aplicações; em contrapartida, 10 estudos não avaliaram suas tecnologias, por serem protótipos ou produtos finalizados que simplesmente não foram aplicados. O Quadro 8 quantifica quantos estudos apresentaram ou não uma avaliação.

Quadro 8. Relação de estudos por avaliação

\begin{tabular}{|c|c|c|}
\hline Avaliação & Quantidade & Relação \\
\hline Sim & 11 & EP1, EP6, EP8, EP9, EP11, EP13, EP15, EP16, EP19, EP20, \\
& & EP21 \\
\hline Não & 10 & EP2, EP3, EP4, EP5, EP7, EP10, EP12, EP14, EP17, EP18 \\
\hline
\end{tabular}

Fonte: Elaborado pelos Autores (2019)

\subsection{QE5 - Em quais níveis de ensino a RA foi usada para lecionar ciências naturais?}

Diante das análises dos estudos primários, destacou-se o ensino médio com um total de 4 aplicações desenvolvidas em RA; o ensino superior e o ensino técnico tiveram, cada uma, um total de 3 aplicações desenvolvidas; no fundamental 2 e 1, somente 1 aplicação foi desenvolvida para ambos. Observou-se que um estudo primário trabalhou com dois níveis de ensino, fundamental 2 e ensino médio, e 10 estudos primários não aplicaram seus métodos em nenhum nível de ensino. A relação de estudos por nível de ensino está no Quadro 9.

\section{Quadro 9. Relação de estudos por avaliação}




\begin{tabular}{|c|c|c|}
\hline Nível de Ensino & Quantidade & Relação de estudos \\
\hline $\begin{array}{c}\text { Nenhum nível de } \\
\text { ensino }\end{array}$ & 10 & EP2, EP3, EP4, EP5, EP7, EP10, EP12, EP14, EP17, EP18 \\
\hline Ensino Médio & 4 & EP6, EP11, EP13, EP20 \\
\hline Ensino Superior & 3 & EP16, EP19, EP21 \\
\hline Ensino Técnico & 3 & EP8, EP9, EP15 \\
\hline Fundamental 2 & 1 & EP6 \\
\hline Fundamental 1 & 1 & EP1 \\
\hline
\end{tabular}

Fonte: Elaborado pelos Autores (2019)

\section{Discussão}

Considerando os resultados apresentados neste mapeamento, destaca-se que na região do Centro-Oeste não há estudos sobre a RA no ensino das ciências naturais. Foi possível observar também que apenas 7 , dos 26 estados brasileiros e um distrito federal, têm pesquisas sobre este tema. Dentre as instituições atuantes em estudos nesta área, a maior fração corresponde às Universidades Federais. Os dados mostram também que a disciplina de química tem menos aplicações de práticas de ensino com RA e que em contrapartida, física é a que mais foi contemplada. Nota-se que, do baixo número de estudos incluídos neste mapeamento, o número correspondente àqueles que foram aplicados é ainda menor, visto que em quase 50\% dos mesmos não constavam avaliações sobre o uso das tecnologias desenvolvidas. Com relação aos outros $50 \%$ dos estudos que foram aplicados, 4 deles foram no Ensino Médio, mostrando assim que os demais níveis de ensino podem ser mais explorados para aplicação do uso de RA.

\section{Conclusão}

Este mapeamento sistemático da literatura foi realizado tendo como base os 21 estudos primários selecionados a partir dos critérios de inclusão e exclusão. Como resultado, identificou-se uma evolução considerável na publicação de estudos sobre RA no ano de 2018; percebeu-se que a disciplina que mais teve aplicação de RA no ensino foi Física; notou-se que há lacunas de pesquisas na região Centro-Oeste; quando investigado a aplicação das tecnologias desenvolvidas pelos estudos, nos diferentes níveis de ensino, foi visto que menos da metade foram aplicadas, mostrando assim que a área de RA ainda pode ser muito explorada no ensino e aprendizagem de ciências da natureza. Como trabalhos futuros, para a continuidade deste estudo, pretende-se expandir as fontes de busca manual e automática, além de incluir estudos primários escritos também em língua inglesa.

\section{Referências}

Associação Brasileira das Empresas de Software (Abes), http://agenciabrasil.ebc.com.br/economia/noticia/2018-08/brasil-ocupa-o-9o-lugarnos- investimentos-em-tecnologia-da-informacao, 2018

Azuma, Ronald T. (1997), “A Survey of Augmented Reality" https://www.mitpressjournals.org/doi/pdf/10.1162/pres.1997.6.4.355 
Billinghurst, M. (2002). "Augmented reality in education". New horizons for learning, 12(5).

Gruber, V., Schaeffer, L., Silva, J. B. \& Restivo, T. (2011). "Model for remote data acquisition and monitoring integrating social media, NTIC's and $3 \mathrm{G}$ cell phone Networks applied to monitoring small wind turbine". Journal of Telecommunications, 7(1), 13-20.

Moro, Roberta G., (2018) "Realidade Aumentada em Livros Infantis: uma revisão sistemática de pesquisas e aplicações”, In: VII CBIE 2018.

Oliveira, Cleber S. de., Mustaro, Pollyana N., (2014) "Revisão Sistemática sobre Jogos Digitais que utilizam Realidade Aumentada no Contexto Educacional Brasileiro", In: III CBIE 2014.

Petersen, K. et al. (2008) "Systematic mapping studies in software engineering". In: EASE.[S.1.:s.n.],v.8,p.68-77.

http://www.robertfeldt.net/publications/petersen_ease08_sysmap_studies_in_se.pd

Petersen, K., Vakkalanka, S., Kuzniarz, L., (2015), "Guidelines for Conducting Systematic Mapping Studies in Software Engineering: An Update". Information and Software Technology, vol. 64, 2015, pp. 1-18.

ProgramaInternacionaldeAvaliaçãodosEstudantes, http://portal.inep.gov.br/web/guest/acoesinternacionais/pisa/resultados, 2018

Schnetzler, Roseli Pacheco. (1992), "Construção do Conhecimento e Ensino de Ciências.", Em Aberto, Brasília, v. 11, n. 55, p. 17-22. 1992. http://emaberto.inep.gov.br/index.php/emaberto/article/viewFile/813/731, Acesso em: 12 jun. 2019 